\title{
Analytical role in clinical toxicology: impact on the diagnosis and treatment of poisoned patients ${ }^{1)}$
}

\author{
Jürgen Hallbach ${ }^{1, *}$, Fritz Degel ${ }^{2}$, Herbert Desel ${ }^{3}$ \\ and Norbert Felgenhauer ${ }^{4}$ \\ ${ }^{1}$ Department Klinische Chemie, Klinikum München, \\ Deutschland (Department of Clinical Chemistry, Munich \\ Clinic, Germany) \\ 2 Institut für Klinische Chemie und \\ Laboratoriumsmedizin, Klinikum Nürnberg, Deutschland \\ (Institute of Clinical Chemistry and Laboratory Medicine, \\ Nuremberg Clinic, Germany) \\ ${ }^{3}$ Giftinformationszentrum Nord, Universitätsmedizin \\ Göttingen, Deutschland (GIZ-Nord Poisons Centre, \\ Germany) \\ ${ }^{4}$ Abteilung für Klinische Toxikologie, Klinikum rechts der \\ Isar der Technischen Universität, München, \\ Deutschland (Department for Clinical Toxicology, Clinic \\ at Technical University of Munich, Germany)
}

\footnotetext{
Abstract

Acute poisoning is a medical emergency situation and requires urgent but adequate medical intervention. The outcome depends on accurate primary care, the correct identification of poison(s) and adequate therapeutic decisions. The present paper reviews the literature concerning the role of analytical toxicology in emergency medicine with particular focus on the last few years. This study is mainly based on a PubMed search: "clinical toxicology review (urine, serum)". Many acutely poisoned patients are treated with no laboratory support other than general clinical chemistry, haemostaseology and haematology. Emergency toxicological analyses that could influence immediate patient management, if offered on the basis of 24-h availability, are most often restricted to ethanol, oximetry and drugs of abuse in urine. Despite paracetamol (acetaminophen) being the top entry on all hit lists of poison control centres worldwide with few exceptions, the availability of its determination in blood on an urgent basis is not standard even at hospitals with

1)Original German online version at: http://www.referenceglobal.com/doi/pdf/10.1515/JLM.2009.021.

The German article was translated by Compuscript Ltd. and authorized by the authors.

*Correspondence: Dr. Jürgen Hallbach, Department Klinische Chemie, Städtisches Klinikum München GmbH, Kölner Platz 1, 80804 Munich, Germany

Tel.: + 49 (089) 30682539

Fax: + 49 (089) 30683911

E-Mail: juergen.hallbach@klinikum-muenchen.de
}

large accident and emergency departments. Therefore, recommendations regarding the assays and methods that should be provided locally and at regional centres were provided for the US and UK, and should be adapted for Germany. Emergency toxicological analyses that could influence immediate patient management are relatively few in number and are remarkably similar worldwide. Recommendations for the US and UK are compared in the present paper together with suggestions for Germany. The first group of these assays should be provided locally by larger hospitals with emergency and intensive care units with a turnaround time (TAT) of $<1 \mathrm{~h}$. Other assays (second group) and a systematic toxicological analysis that can help improve patient management after a period of primary stabilisation of vital functions and general supportive therapy can be provided from regional centres with a TAT of $4 \mathrm{~h}$. The need for such centres and the repertoire of tests and methods that should be available will be adapted and discussed. It is well known that comprehensive toxicological analysis incorporating various methods can identify much more substances than are clinically suspected. At the same time, this information might have no clinical utility owing to the time required for sampling, transportation, analysis and reporting, or because the toxicological report might be inconsequential. This has contributed to a range of clinical opinions and practices, from a minimalist approach to a "shotgun" approach of broad-based laboratory testing. The present review should help to understand and discuss the pros and cons of both approaches.

Keywords: analytical toxicology; chromatography; emergency medicine; toxicological centres.

\section{Introduction}

Acute poisoning is a frequent cause of emergency hospital admission. Many intoxicated patients recover completely without specific treatment [1], while in more severe cases a toxicological analysis can be decisive.

Severe acute poisoning is an internal-medical emergency requiring quick and targeted action. Progression and prognosis depend crucially on the correct emergency primary care, determination of the poison involved and the proper treatment measures. Severe poi- 
soning is characterised, for example, by a coma, which may be present primarily, or set in later. There are a variety of causes for sudden coma (Figure 1). Environmental factors and/or external medical history can also point to poisoning.

From a clinical perspective, the toxicological analysis in the context of the clinical strategy of suspected poisoning plays a recognisably pre-eminent role. Proudfoot has postulated the following approach to clinical management [2]:

- Clarifying the need and possibly implementing lifesustaining measures

- Confirmation of the diagnosis of poisoning by identifying the poison(s)

- Introduction of therapeutic measures against toxic effects on organ functions

- Prognosis regarding progression and expected outcome of the poisoning, and evaluation of the potential psychiatric significance of the event

As early as four decades ago, Arnold [3] described the co-operation between the toxicology laboratory and intensive care medicine. In 1972, Free and Free [4] crit- ically discussed this, and in 1974 the first major overview was published in the journal Clinical Toxicology [5]. Nearly 10 years later, the founder of modern toxicological analysis, Irving Sunshine, defined the role of the toxicology laboratory in emergency medicine [6, 7].

Recent recommendations for the organisation of toxicological analysis were issued for the US by the National Academy of Clinical Biochemistry NACB [8], for Britain by Flanagan [1] and for Germany, in the form of relevant chapters of a manual $[9,10]$. This paper discusses these recommendations, i.e., their pros and cons, taking into account other relevant literature.

Qualitative tests on blood and urine are useful to prove ingestion of toxins, while quantitative tests to determine an appropriate therapy for certain toxins, such as paracetamol, salicylates, and paraquat, are indispensable [11]. The more extensive the toxicological analysis, the more clinically unsuspected substances can be detected. Often, the information from the clinical and toxicological findings does not have any direct benefit because the time needed to transfer results is too long or because the analytical results are not accompanied by clinical consequences [8]. This has led to two extreme opinions in

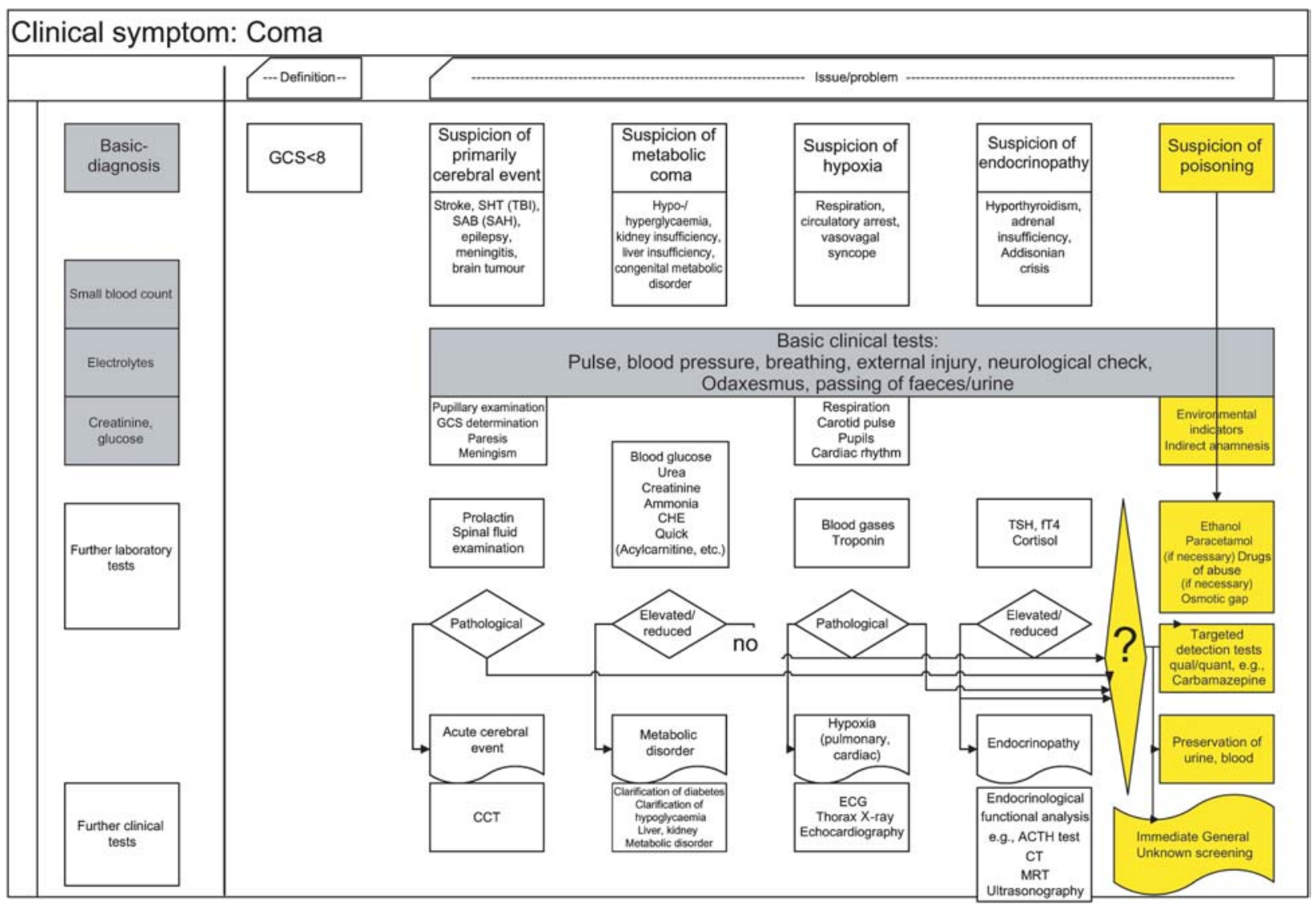

Figure 1 Procedure for unclear poisoning or differential diagnosis of an unclear coma.

"General Unknown" screening, provided only by certain centres, can be preceded, on site, by tests for common noxious substances, particularly ethanol, paracetamol and drugs of abuse. In addition, special clinical-chemical tests, e.g., osmotic gap, may be helpful. This is also true of targeted tests in the area of TDM. GCS, Glasgow coma scale; SHT, Traumatic brain injury (TBI); SAB, Subarachnoid haemorrhage (SAH); CCT, Cranial computer tomography; TDM, Therapeutic Drug Monitoring. 
clinical practice. They range from a minimalist to a "scattershot" approach, with a very varied analysis program. This overview is intended to make these positions better understood and to discuss them.

\section{Incidence of poisoning}

Exposure to drugs and toxins is a common cause of medical emergencies that end up in the emergency department [8]. In emergency medicine, there is always a need for quick decisions and always a shortage of reliable information that support these decisions. This is especially true of intoxicated patients.

The range of possible poisons is very diverse and includes drugs, legal and illegal drugs, household products and chemicals of any kind, cosmetics, pesticides, insecticides and rodenticides, as well as plants, fungi and animal poisons. The most frequent queries sent to poison information centres both in the case of adults and children are related to poisoning with pharmaceutical drugs. In the case of children, there are also queries mainly about plants (yew, plants of the nightshade family, laburnum, hogweed, garden beans). In detail, for example, the following query statistics have emerged (Poison Information Centre Munich): $25.6 \%$ medicines, $15.5 \%$ consumer products (cosmetics, household items, etc.), $14.1 \%$ plant poisons, $9.1 \%$ chemicals, $7.9 \%$ animal toxins, $6.8 \%$ foodstuffs, $6.2 \%$ drugs of abuse, $5.5 \%$ pesticides, $3.9 \%$ solvents, $2.7 \%$ gases, $2.1 \%$ detergents and cosmetics.

Most intoxications in adults are caused by alcohol and medications or a combination thereof. Among medications, hypnotic drugs, sedatives, psychiatric medications and analgesics have been most prominent for a long time. In $70-90 \%$ of treated cases of poisoning involving adults, the cause is attempted suicide, with the group aged 18-40 being most prominent. A relatively frequent cause in adults is drug intoxication. In children, especially in infants up to age six, poisoning is almost always the result of an accident (approximately 99\%); due to a lower dose of poison, their condition generally runs a milder course. Other, rare, causes include side effects of medicines, medical accidents, accidental poisoning or the Munchausen Syndrome by proxy. School children up to age 14 are also the most common victims of accidental poisoning $(85.6 \%)$. The current figures are available on the internet (www.toxinfo.org/publikationen/Jahresbericht).

Based on estimates, Germany, for example, treats over 200,000 inpatients for poisoning every year. This corresponds almost exactly to the number of patients treated for acute myocardial infarction. Unfortunately, according to ICD-10 S00-T98 injuries, poisoning and certain other consequences of external causes are combined.

Poisoning treatment has changed drastically in the last decade by largely forgoing detoxification measures. But in severe cases of poisoning, especially with membrane- stabilising substances and calcium channel blockers [12], a very aggressive drug treatment and in some cases even an extracorporeal organ support therapy are the approaches of choice. The need for toxicological analysis with such an approach seems self-evident.

\section{Methods of poisoning analysis}

Starting in the 1940s and 1950s, analytical toxicology underwent a rapid technical development driven by spectroscopy and thin-layer chromatography [13].

Preserving evidence For any suspected case of poisoning, suitable material must be secured as evidence, without contaminating it, to show poisoning. Securing evidence and toxicological analysis help to identify poisons and thus to secure the diagnosis and risk assessment concerning the likely course of the poisoning. Most common evidence includes urine samples $(10 \mathrm{~mL})$ and serum or plasma (at least $3 \mathrm{~mL}$ ), and possibly vomit, food and fungal remains, empty boxes of medicines, syringes and other objects associated with the poisoning. The evidence thus secured must be marked with instructions identifying the type of material, the time of sample collection and a unique patient identification number. If the patient requires an antidote, blood and urine samples should be drawn before the administration of the antidote, as it may interfere with the poison analysis. The analysis of stomach contents, only rarely available because of the risks of gastric lavage, can usually be waived, nor is it any longer specifically recommended for patient management [8]. In special cases such as newborns, an investigation into rather unusual sample materials such as meconium may be important [14].

A so-called clinical-chemical base program (Table 1) also plays an important role in intoxication in connection with the initial lab-based diagnostic assessment of the patient's situation.

Frequently used methods of systematic toxicological analysis (STA) and their limitations are:

- HPLC-UV/vis-spectrometry (HPLC-DAD). Here, the foreign substances to be analyzed are transferred to an organic solvent mixture following sample extraction, chromatographically separated and identified by retention time and UV/vis-spectra. Limiting factors are the separation efficiency and the often non-specific UV spectra of the foreign substances. REMEDI-HS, which is relatively widespread, has been used for this purpose so far, with TOX.I.S [15] being a possible alternative. For parallel tests, run by way of an example, to detect amphetamines, cocaine and opiates in 405 cases, the agreement with GCMS was $80 \%$ for TOX.I.S and $78 \%$ for the REMEDI-HS.

- GCMS (gas chromatography-mass spectrometry). This is the current standard procedure with high selectivity and highly accurate identification through comparison of mass spectra [16-20]. For the comparison 
of spectra, very large databases with entries for up to 300,000 compounds are used. The GCMS can be expanded to include methodological variations, if necessary, to increase the sensitivity and specificity [21]. This is limited by the fact that the substances to be detected must not be too polar or too "large". This limitation can be only partially offset, e.g., by special derivatisation procedures.

- HPLC(LC)-mass spectrometry, e.g., LC-MS/MS, LCMS-TOF or LC-MS-QTrap. HPLC-mass spectrometry methods are increasingly used in toxicological analysis. Detailed information on the principle, methodological implementation, details on the interfaces between LC and MS, on practical examples and on the validation of LC-MS methods have been available in the literature for some time [16, 17, 19, 20, 22, 23].

- If the laboratory also conducts metal analysis in poisoning cases, the method of choice is ICP-MS. Since metal analyses are less urgent than others, other

Table 1 Basic clinical-chemical parameters in poisoning cases.

\begin{tabular}{|c|c|}
\hline Parameter & Meaning \\
\hline Erythrocytes & $\begin{array}{l}\text { For example, recognition of toxic haemolysis } \\
\text { (free } \mathrm{Hb} \text { ) }\end{array}$ \\
\hline Leucocytes & Stress leucocytosis in intoxication cases \\
\hline Thrombocytes & Recognition of consumption coagulopathy \\
\hline Quick or INR & $\begin{array}{l}\text { Dysfunction of blood coagulation, e.g., } \\
\text { coumarin intoxication }\end{array}$ \\
\hline РТT & Dysfunction of blood coagulation \\
\hline D-Dimer & Recognition of consumption coagulopathy \\
\hline Sodium & Electrolyte imbalance \\
\hline Calium & Electrolyte imbalance \\
\hline Chloride & Electrolyte imbalance \\
\hline Calcium & Electrolyte imbalance \\
\hline Glucose & Clarification of hypo- or hyperglycaemia \\
\hline Urea & Recognition of acute kidney failure \\
\hline Creatinine & Renal function, low sensitivity \\
\hline Cystatin C & Renal function, sensitive \\
\hline ASAT & Recognition of ubiquitous cell damage \\
\hline ALAT & $\begin{array}{l}\text { Liver involvement (e.g., paracetamol } \\
\text { intoxication) }\end{array}$ \\
\hline GGT & Liver involvement \\
\hline $\mathrm{CK}$ & Co-involvement of muscles \\
\hline $\mathrm{CHE}$ & $\begin{array}{l}\text { Recognition of insecticide and combat } \\
\text { agent poisoning }\end{array}$ \\
\hline Troponin & Cardiac co-involvement \\
\hline Anion gap & $\begin{array}{l}\text { Recognition of acidic toxic metabolites, } \\
\text { e.g., oxalate (glycol intox.) }\end{array}$ \\
\hline Osmotic gap & $\begin{array}{l}\text { Recognition of low-molecular toxins, } \\
\text { e.g., methanol }\end{array}$ \\
\hline Prolactin & Ruling out a cerebral event \\
\hline $\mathrm{pO}_{2}$ & Assessment of oxygenation \\
\hline $\mathrm{pH}, \mathrm{pCO}_{2}, \mathrm{BE}$ & $\begin{array}{l}\text { Recognition of acidosis, e.g., salicylate } \\
\text { poisoning }\end{array}$ \\
\hline Lactate & Recognition of anaerobic metabolic status \\
\hline $\begin{array}{l}\text { Urine test } \\
\text { strips }\end{array}$ & Recognition of kidney damage, acidosis \\
\hline $\begin{array}{l}\text { Urine } \\
\text { sediment }\end{array}$ & $\begin{array}{l}\text { Recognition of kidney damage, } \\
\text { crystallisation }\end{array}$ \\
\hline $\begin{array}{l}\text { Alpha1- } \\
\text { microglobulin/U }\end{array}$ & $\begin{array}{l}\text { Recognition of kidney damage (proximal } \\
\text { tubule) }\end{array}$ \\
\hline
\end{tabular}

transport routes on longer distances are acceptable here as well.

Overall, a proven approach taken by many laboratories is the parallel application of GCMS and HPLC methods, e.g., REMEDI [24, 25] or HPLC-DAD. HPLC-MS and, in particular, HPLC-tandem MS (HPLC-MS/MS) are even better at identification and more sensitive, and are ideal for quantifications.

Other new opportunities for a broad-based screening, also with greater sensitivity, can be found in the use of the hybrid triple quadrupole-linear ion trap mass spectrometers (QTrap) and time-of-flight mass spectrometry (TOF):

QTrap: This enables a so-called multi-target screening of currently 300 substances in a chromatographic run [26], although its extension to more than 1000 substances is anticipated. Identification accuracy is significantly increased by simultaneous detection (MRM survey scan) and information-dependent acquisition (IDA) in the third mass spectrometer that functions as an ion trap. An automatic database search is done in an MS/MS library that is based on EPI spectra for three different collision energies.

HPLC-MS-TOF: Recently, for example, a method for drug detection in hair was published [27]. The identification of toxic substances is based on the TOF analysis of up to four independent variables: exact mass, isotope ratios (I-fit), retention time and fragment analysis. The three mass spectrometry parameters are highly reproducible and, unlike HPLC-MS/MS, not, or at least only slightly, device-dependent. The example of psychoactive substances has shown that HPLC-MS-TOF allows for the identification of metabolites in authentic samples even without corresponding reference substances [28].

All quantitative procedures require validation, with at least six to eight concentrations above the measuring range with matrix-containing samples to verify the validity of the calibration curve. In addition, certified control samples or control samples prepared independently of the calibration standards (low, medium, high) must be used, and participation in inter-lab tests, where available, is mandatory [1].

\section{Drug-screening procedure}

For the detection of drugs in urine, immunoassays are frequently used as a first step. There are group-specific and substance-specific tests, test strips and mechanised methods.

Cut-off values In particular the group-specific immunoassays are always subject to the risk of cross-reactivity, which may lead to unexpected, often false positive, results. The definition of positive/negative is determined on the basis of the respective cut-off value. The cut-off is a variable determined purely by convention [8].

A value above the cut-off is rated positive. Values just below the cut-off value can be regarded as "borderline 
negative" and values just above as "borderline positive". In the strip test, the user cannot change the cut-off. The cut-off value is usually significantly higher than the detection limit of immunoassays. The definition of this numerical value is governed by both analytical and strategic factors (e.g., for reasons of "drug policy"). It is to follow the requirements of the person/institution requiring the analysis and the cut-off must not be set too high [8]. There are three problem areas of immunological drug tests, which have yet to be solved adequately: dependence of the cut-off values on the problem posed, uniform use of such cut-off values, cross-reactivity and detection of conjugated metabolites.

Group-specific immunoassays Amphetamines and similar compounds are detected in different tests to varying degrees. Barbiturates hardly occur anymore today. Benzodiazepines may have a glucuronidated hydroxyl group (e.g., lorazepam) and may then be captured only following a glucuronide split. Opiate tests capture the heroin metabolite MAM (monoacetylmorphine), morphine, codeine, dihydrocodeine, etc., but not opiate-like substances (fentanyl, tramadol and others). After consuming poppy seeds, opiate tests can yield positive results for the first few hours.

Substance-specific immunoassays Specific MAM test for heroin, THC for cannabinoids, methadone or metabolite detection (EDDP), cocaine or metabolite detection (benzoylecgonine) produce more specific responses. Still, positive immunoassay results should always be considered preliminary until they are confirmed by chromatographic methods [8].

Manipulations Urine is easily manipulated, so the sample must be collected under supervision, and procedures for detecting manipulations should be used (e.g., temperature, creatinine, osmolality).

Confirmatory analysis This is not as explicit a requirement in clinical-toxicological analysis as it is in forensic practice, but unconfirmed findings must be clearly labelled as such.

Preservation of samples Remaining samples after the analysis must be stored properly and safely for a defined period of time [1].

\section{Strategies}

The clinical approach to the poisoned patient begins with the analysis of the external circumstances and the search for toxidromes. This refers to a common characteristic constellation of symptoms and findings. The expectation of the clinician [29] is that the analysis detects the toxins, confirms or rules out poisoning, indicates the severity and assists in the follow-up.
The term "toxicological screening" (drug screen) is very misleading, because it ultimately implies a screening for all conceivable substances, which is generally not possible [30]. It should therefore, be avoided.

More than half of all cases of poisoning today involve mixed intoxications whose diagnosis and prognosis regarding the course of the condition are hardly possible on no more than clinical aspects without demonstrating the presence of the poison(s). It should also be considered that acute intoxication can hide behind an initially confusing set of clinical symptoms, or it may be a secondary aspect of an emergency admission to hospital, e.g., after accidents, burns, etc.

While in cases with objective suspicion of intoxication targeted toxicological laboratory examinations, the consultation of poison information centres and treatment in specialised facilities can quickly lead to appropriate therapy, in the other cases mentioned, one must reckon with a not inconsiderable number of cases where intoxication goes unrecognised or is discovered late or too late. To avoid this, a toxicological investigation in the form of a "general unknown" search, i.e., a comprehensive toxicological screening, should be conducted in any vague, acute disease situation. However, this is impractical not only for cost reasons. Hence, it seems reasonable to order a toxicological analysis on the basis of defined criteria and suspicious factors (Figure 1).

Caution is warranted when laboratories focus exclusively on detecting toxidromes [8], because it can easily happen that the requirement of an important toxin proof is overlooked.

Overall, toxicological laboratory investigation should be done for all suspected cases of poisoning, and for all diseases, where intoxication cannot safely be ruled out as a cause. This is particularly true for diseases with vigilance impairment that is not explained by a neurological disorder, a metabolic or endocrine disorder. Particularly important are toxicological investigations on poisonings that are preceded by a symptom-free interval before the onset of irreversible organ damage (e.g., paracetamol and amanitin intoxication). In these cases, however, specific therapy must generally start as soon as there is suspicion, prior to knowledge of the toxicological test results. The therapy may be modified where appropriate and, in the presence of a negative examination result, aborted [9, 10].

Furthermore, toxicological analyses should be mandatory for all severe intoxications in which extracorporeal detoxification methods are considered. In carrying out an extracorporeal detoxification procedure, it makes sense to determine blood concentration levels prior to, and in reasonable intervals during the procedure, immediately afterwards, and about four hours after its completion. This allows monitoring of the elimination kinetics of the noxious substances from the plasma and helps to determine optimal duration of extracorporeal therapy. By measuring the concentration of the poison after about four hours after termination of the therapy, it is possible 
to identify a rebound phenomenon by redistribution from tissue compartments [9, 10].

The toxicology laboratory will be called upon in connection with the following problems or questions [9, 10]:

- Exclusion/confirmation of the suspected diagnosis of "poisoning"

- Prognosis regarding progression and possible outcome of the treatment

- Monitoring of therapy

- Brain death diagnosis

- Confirmation of alcohol, drugs or medication abuse

- Detoxification and withdrawal treatment

This results in the following tasks for the laboratory $[9,10]$ :

- Evidence of probable poisoning, involving more or less reliably known substances

- Tests in the event of a clinically highly probable suspicion of poisoning, without reference to specific substance(s)

- Exclusion of poisoning through differential diagnosis

- Quantitative tests to monitor the course of therapy

- Quantitative laboratory tests as part of the brain death diagnosis

- Tests to exclude/confirm drug abuse

A "general unknown" analysis in the shortest possible time is the real challenge for the clinical toxicology laboratory. To limit one's examination to only the suspected toxins is risky, because this assumption is often not true and the (impermissible) finding "Toxicology Negative" can easily give the treating physician a false sense of security, who then discards the suspected diagnosis of "poisoning". However, since even an extensive screening can never test for all possible poisons, the findings report should address in as much detail as possible the substances that can be ruled out at least in toxicologically relevant concentrations, and the substances that cannot be detected with (sufficient) certainty. Here, the investigating laboratory should provide competent assistance when it comes to enlisting a specialist laboratory and with respect to questions concerning pre-analytics.

Ideal conditions for the clinical and toxicological analysis are rather rare, and local conditions and thus the locally established strategies show significant differences. These various strategies, however, have in common that in toxicological investigative analysis both different test materials (urine, blood, etc.) and various investigative techniques must be used $[9,10]$. Chromatographic techniques, in particular, GC-MS and HPLC-DAD (or mass-) spectrometry, are most prominent in this regard. We recommend either the combination of several GC-MS or HPLC runs (sample preparation, derivatisation, column selection and separation conditions) or the combination of both techniques [24]. The decisive factors are inter alia the equipment as well as the experience and training of the staff with respect to the techniques employed.
If one assumes that for the clinician it is particularly important to focus on such substances that require specific treatment for the patient [31], then one must also discuss, as an alternative to the primarily qualitative STA, whether patients in emergency care with suspected acute intoxication should primarily be subjected to a defined program of quantitative analyses. As part of such an approach, 351 serum and 39 urine samples were tested in parallel for 80-90 substances in a matter of $24 \mathrm{~h}$ [32]. In this case too, early dialogue between clinicians and toxicologists is a prerequisite for a rational use of the programme provided.

\section{Clinical toxicological interpretation}

During the clinical interpretation of toxicological findings, possible differential diagnoses of the patient must be reviewed critically. First, one must clarify whether the patient is intoxicated. It may involve the taking of a therapeutic dose of a medication in the context of self-medication for an acute internal or neurological condition. One example is the detection of salicylic acid in a now comatose patient who, because of a sudden headache brought on by subarachnoid haemorrhage, took a therapeutic dose of acetylsalicylic acid. A quantitative determination in this case facilitates the diagnosis. It also must be clarified whether this symptom complex can be explained primarily by an intoxication or whether secondary complications, e.g., caused by hypoxia, determine the clinical picture. To answer these questions, knowledge of the patient's case history, clinical examination findings, quantitative analysis of noxious substances, clinical-chemical studies, neurological observation and possibly instrumental tests, such as an EEG, cranial computer tomography and/or NMR or carotid angiography, can help $[9,10]$.

Given the urgency of the findings, the results and their interpretation are typically communicated by phone in advance. This approach should also be subject to standardisation and quality management [1].

Severe intoxication can often lead to metabolic and endocrine imbalances or coagulation disorders that can shape the further clinical picture overwhelmingly.

The following points must be considered in detail $[9,10]$ :

- In terms of differential diagnosis, what diseases could be represented by the present symptoms?

- In addition to the poisoning, is there an internal or neurological condition?

- Can the toxins or their metabolites or another illness influence the toxicokinetics (e.g., slowed-down gastrointestinal action in combination with anticholinergics)?

- Does the clinical picture fit the effect profile of the detected substances? 
- Is the metabolism affected by shock, liver or kidney insufficiency?

- In the case of mixed intoxications, are there any possible interactions (antagonism, synergism) with the detected substances that should be taken into account?

- Do genetic polymorphisms influence the pharmacokinetics/toxicokinetics?

- Does enzyme induction influence the pharmacokinetics/toxicokinetics?

- Is there a risk of long lasting sequelae?

- Which therapeutic consequences (e.g., antidote therapy, enhanced poison elimination) derive from the results of toxicological analysis?

This yields important information for therapeutic conclusions. The therapeutic decisions of the clinician take into account both the toxicological-analytical findings and the clinical symptoms of the patient. Occasionally, the laboratory findings and the clinical findings do not match. For example, this may be the case in the early phase of intoxication with substances that lead to clinical symptoms (e.g., such as poisoning with paracetamol) with some delay, or in the late phase, in which irreversible damage has already occurred and the substance has been excreted (e.g., CO poisoning). In such cases, it is particularly important that all possible causes for the discrepancy between clinical and laboratory findings be discussed by the clinician and analyst $[9,10]$.

The results of immunochemical testing must include clear references to the most important cross-reactivities, cut-off values and other restrictions (8). In emergency care, attention must be paid to the use of cut-off values that should be as low as possible so as not to overlook any substance [8]. The use of serum/plasma instead of urine has two important advantages: Unlike urine, blood contains unconjugated compounds which are easier to detect, and results from serum/plasma correlate better with the current situation of the patient. However, many substances or metabolites in the urine usually occur in much higher concentrations and can thus be detected more reliably.

When findings of toxicological analysis are communicated, this is often followed very quickly by the question of "how much", that is to say, the issue of quantification. However, blood concentrations are only needed if treatment depends on the results [29].

\section{Necessity and extent of toxicological analysis from a clinical perspective}

While we are virtually flooded in the literature with studies on new methods, there are only few that deal with the understanding of the clinician to employ these techniques, but none that looks objectively at their usefulness and cost-effectiveness [30]. It is precisely in this context that the need for and in particular the extent of toxicological analysis is viewed critically by some experts in clinical emergency medicine. It is frequently noted that toxicological analysis cannot, and must not, replace the careful clinical diagnosis and clinical management of a poisoned patient [33]. The use of the laboratory should be preceded by critical reflection and the laboratory tests should be limited to what contributes directly (and immediately) to the appropriate clinical management of the patient [33]. This requires ongoing communications in order to optimise resources. This view has become very important, of course, especially at the present time. There is a lack of consent yet which tests contribute to the clinical management and what turnaround times are required.

On the other hand, there has been a rough assumption for some time that, with the help of the patient's medical history and clinical survey, correct conclusions about a substance overdose can be drawn in only $50 \%$ of cases. This is confirmed by the observation of Tournier that an intentional substance overdose - in an emergency setting as compared to limited toxicological tests (cannabis, opiates, buprenorphine, amphetamines, cocaine, LSD) - can be diagnosed through the patient's medical history and clinical examination only in $50 \%$ of cases [34].

Also, a further current study has found, after analysing 947 cases with acute intoxication, that there is a $70 \%$ match between clinical suspicion and analysis only as far as ethanol and paracetamol are concerned, whereas for all other substances tested (a total of eight serum tests), that match was below $51 \%$. The authors therefore, concluded that the reliability of the clinical diagnosis varies so much, and that therefore, tests should be conducted for all substances which are crucial to deciding on specific treatment [31].

Especially in the paediatric literature, there are several critical studies and opinions, such as those by Sugarman et al. [35], who, in a paediatric emergency situation, evaluated the documentation of 338 patients with suspected poisoning and compared them to the results of a toxicological screening (194 serum and urine, 44 serum and 95 only urine). Only 22 patients were identified for unexpected results and, finally, the clinical management was modified on the basis of the toxicological analysis only for three patients. Because of the very low frequency of unexpected results that he observed, he recommends that emergency doctors should re-evaluate the indication for toxicological screening in paediatric patients carefully. Similarly, Belson and Simon [36] has carried out a study and compared the results of a very limited range of tests (in the serum: ethanol, aspirin, paracetamol; in urine: benzodiazepines, barbiturates, amphetamines, cocaine, opiates and phencyclidine) to HPLC-screening. All positive findings (234 of 463 cases) were categorised according to the doctor's findings in terms of "yes/no", "suspected because of medical history and clinical examination yes/no" and "clinically significant yes/no". Only $3 \%$ of the cases yielded clinically significant findings on the basis of HPLC alone, but none resulted in any changes to clinical management. It was concluded 
that the limited testing programme was sufficient and that the additional HPLC test would be costly without producing any clinical benefit [36].

In special cases, such as sudden infant death [37], however, toxicological analysis is essential to ruling out, e.g., a Munchausen by proxy syndrome or killing by poison.

In the field of drug analysis, however, the contributions made by laboratory testing are considered very important especially in connection with adolescents [38]. But here too emphasis is given that laboratory tests are only one important aspect that cannot replace the ongoing therapeutic alliance of the attending physician with the patient.

\section{Diagnostic pathways}

Position papers on the clinical management of poisoned patients exist for many issues, e.g., "alkalinisation of urine" [39], whereas, apart from the opinions discussed in this paper from the US [8], UK [1] and the manuals of Külpmann $[9,10]$, there is very little literature so far on an efficient lab-diagnostic approach. As part of an activity of DGKL (German Society for Clinical Chemistry and Laboratory Medicine) led by W. Hofmann (Munich), a working group is to propose "diagnostic paths" in toxicology [40].

First, the working group has dealt with the topic of paracetamol poisoning and developed a preliminary fivestep strategy: 1) Assessment of exposure. 2) Risk assessment. Here no further action is required if the dose is below $150 \mathrm{mg} / \mathrm{kg}$ of the body weight, if no other toxicologically relevant substances were ingested and if there is no suicidality. 3) Symptom-based therapy. Measurement of basic clinical-chemical parameters (see Poisoning with Paracetamol) and determination of paracetamol levels. 4) If necessary (see chart according to Rumack), antidote therapy with acetyl cysteine. 5) Monitoring progression with ALT, Quick (INR), bilirubin and creatinine.

\section{Hospitals tests in acute care}

Gibitz presented a programme of quick toxicological tests as early as 1981 [41], which can serve as "preliminary analysis" in the laboratory of an acute care hospital. The need for such simple tests, which can also be used in smaller hospitals, was also postulated by Flanagan et al. [13]. An automated analysis programme on a clinical-chemical analyser with serum/plasma as samples was then proposed in a publication in 1991 [42]. This methodology still exists as a basic programme despite several technical advancements. Such automated procedures do meet the requirement of having a turnaround of less than an hour for emergency care, but the test configuration and choice of analytes is more often gov- erned by concerns of "workplace-testing" or drug analysis than concerns of emergency medicine involving acute intoxication [30].

There is the view that toxicological emergency analysis is possible only with specific and rapid methods, which can be achieved by chromatographic methods only, while immunoassay screening has been discarded as useless [29].

However, the resources available in typical hospital laboratories are usually limited and, therefore, comprehensive toxicological analysis in real time and on site is generally not possible [8] - except for hospitals with a specialist laboratory. Therefore, the NACB has established recommendations for rapid tests in hospital laboratories [8]. Selection is based on clinical relevance, availability of tests and the direct impact of the test results on clinical management or patient care.

These recommendations for quantitative emergency analysis of serum/plasma, which any major hospital with emergency medical care should be able to turn around within one hour, have been summarised in Table 2. These should be supplemented by qualitative urine tests (Table 3). Many emergency physicians, however, do not trust immunochemical urine drug testing [8], which may particularly concern test strips. At least ethanol, paraceta-

Table 2 Recommendations for quantitative toxicological tests with a turnaround time of less than one hour.

\begin{tabular}{lcc}
\hline Toxin & NACB (US) & Flanagan (UK) \\
\hline Paracetamol & + & + \\
Lithium & + & + \\
Salicylate & + & + \\
Oximetry (COHB, MetHb) & + & + \\
Theophylline & + & + \\
Valproic acid & + & - \\
Carbamazepine & + & - \\
Digoxin & + & + \\
Phenobarbital & + & - \\
Iron & + & + \\
Transferrin & + & - \\
Ethanol & + & + \\
Methanol & $+^{*}$ & $* *$ \\
Ethylene glycol & ${ }^{*}$ & $* *$ \\
\hline
\end{tabular}

${ }^{*}$ If necessary, via the regional toxicological centre. ${ }^{* \star}$ Less urgent.

Table 3 Recommendations for qualitative toxicological tests with a turnaround time of less than one hour.

\begin{tabular}{lll}
\hline Toxin & NACB (US) & Flanagan (UK) \\
\hline Cocaine & + & - \\
Opiates & + & - \\
Barbiturates & + & - \\
Amphetamines & + & - \\
Propoxyphene & $(+)$ & - \\
Phencyclidine & $(+)$ & - \\
TCAs & $(+)^{\star}$ & - \\
Paraquat & - & + \\
\hline
\end{tabular}

${ }^{*}$ Due to analytical non-specificity. 
mol, salicylates, $\mathrm{COHb}$, cholinesterase, iron, lithium and digoxin should be measurable in emergencies [8]. The NACB has declared the following substances specifically to be irrelevant to emergency medicine: THC, LSD, methaqualone, ibuprofen and cotinine [8].

For specific patient groups, such as in paediatrics, these recommendations must obviously be adapted. Also, e.g., illegal drugs, the preferred kind consumed by adolescents, differ in their frequency distribution from that in adults. Of particular importance are THC, ecstasy, and also gamma-hydroxybutyric acid (GHB) as well as the corresponding lactone $(\mathrm{GBL})$, both of which are known as "liquid ecstasy".

National or regional differences arise, for example, from the prevalence of various toxins. Thus, paraquat exposure is very rare in the US (only 120 cases in 1998, none fatal).

\section{Regional laboratory centres}

A broad toxicological testing programme is usually not required for asymptomatic patients or patients whose condition significantly improves while in the emergency department. The Guidelines of the National Academy of Clinical Biochemistry and Laboratory Medicine [8] recommend for all comatose or coma-developing patients a broad screening for the detection of substances with clinical relevance that cannot be captured by local rapidtest screening. This analysis is usually done in a regional centre with good technical equipment and specially trained staff. The examination requirement should follow the stabilisation of the patient and be in consultation with the poison centre.

However, to assess the risk to the patient more specifically and to confirm the diagnosis of suspected intoxication, a comprehensive toxicological analysis would be desirable also in cases of less severely intoxicated patients. Comprehensive toxicological analysis is also referred to as systematic toxicological analysis (STA) or "general unknown screening". Only with very few specific issues is it sufficient to rely primarily on the detection of some selected toxic substances. These are mostly cases in which a certain toxic substance is expected with a high probability and where the substance occurs in a toxic quantity, which requires immediate specific therapy. In these cases, because of the clinical decision for or against appropriate therapeutic measures, it is necessary immediately to quantify the toxic substance (also see Table 2). In general, however, implementation of STA as a broad-based chromatographic and primarily qualitative investigative analysis on blood and/or urine samples will be necessary. Today there are different chromatographic procedures, which are obtainable from specialised laboratories at all times. The nearest suitable laboratory can be located by calling the (next) poison centre. In general, transport time for samples may be up to two hours, and processing may also take another two hours. Therefore, the first few hours in the event of intoxication must be bridged with measures aimed purely at symptoms and primarily with general life-sustaining measures until more specific treatment measures can be taken on the basis of toxicological analysis results, and if necessary, after consultation with the poison centre. The time required for the entire process (sampling, transport, analysis and diagnosis) is critical in terms of possible clinical decisions and should be evaluated accurately and optimised where possible.

Proof of poison is indispensable not only for the decision regarding specific therapeutic measures, but also for the case documentation. The need for quantitative testing can be clarified in dialogue with the laboratory. Ruling out poisoning is also extremely important for the purposes of differential diagnosis. However, even an extensive negative STA is no guarantee that no foreign substances actually exist, especially since quite a few relevant toxins are not detected in normal STA test processes, requiring further targeted special tests (e.g., diquat, amantoxins, bromides).

A special area of clinical-toxicological analysis is the detection of substances depressing cental nervous functions as part of the process of determining brain death [1]. Brain death-investigation records allow for the early determination of death in patients using life-sustaining support systems such as mechanical ventilation [43]. Centrally active drugs, particularly barbiturates (e.g., thiopental) and benzodiazepines (e.g., midazolam), have an inhibitory effect on respiration and affect clinical apnoea testing, which is why, the presence of these drugs in an effective concentration must be ruled out by means of validated analytical methods [44]. The correct interpretation of the results, in turn, requires close collaboration between toxicologists and clinicians [43].

Flanagan [1] emphasises that a regional specialist toxicological laboratory must have appropriate instrumental equipment, reference materials, trained personnel, detailed work rules (SOPs) and quality management system. It is obvious that such specialist laboratories should be part of or be set up in close proximity to an existing hospital laboratory [1]. In such a case, many of the necessary facilities are in place and the staff is trained to deal with potentially infectious samples.

Because of the high degree of specialisation in clinical analytical toxicology, it is necessary that such a specialist laboratory receive a "critical mass" of testing orders and additional financial support $[1,8]$. Additional tasks in the field of drug analysis, special therapeutic drug monitoring or metal analysis can then be handled. In one of the author's hospital's own laboratories, as concerns the field of toxicology, all chromatographic tests for the department are carried out, given their methodological similarities, e.g., hormone and vitamin testing. To operate a regional centre in an economically viable manner, one might consider also to include environmental analysis, exposure analysis and biomonitoring $[45,46]$. However, it should be ensured that the clinical toxicological expertise is quite important. From this point of view, integration 
into an institute of laboratory medicine is probably the cheapest way.

Sources of information and professional staff development Important sources of information can be found, for example, on the internet (Table 4). Not only the staff of specialist laboratories requires constant training and professional development in instrumental analysis and clinical toxicology, but also the staff that conducts tests at the local laboratories, and last but not least, emergency physicians who use the service must be instructed in the testing programme and its limitations, sampling, transport of samples and the interpretation of results [1]

\section{The importance of poison information centres}

Poison information centres can provide an important aid in the therapeutic conclusions drawn from toxicological and clinical findings. They can provide diagnostic recommendations, assess the risk of poisoning, give treatment recommendations, and document all cases. Typically, more than 1000 SOPs and databases (Poisindex, Toxbase, Toxinfo, GIZINDEX) and extensive specialised literature can be accessed during consultation. At least in all ambiguous cases and especially in cases of poisoning with rarer toxic substances, the centres should be contacted for further advice. The reporting to the poison centre of a critically analysed final assessment of a poisoning case by clinicians and analysts can contribute to improve those datasets, especially in comparisons with other cases (transversal interpretation). Especially in cases of intoxication involving rare toxins, a careful critical documentation and dissemination of experiences can be greatly beneficial for the treatment of other cases of poisoning. The poison information centres can provide substantial support for the specialist laboratories by supplying local epidemiological data, through their information policy and information on appropriate methods and testing procedures [1].

\section{Significance of common and main poisons}

Sedative poisoning Intoxication with sleeping medication is observed with suicide attempts or accidental intoxications among drug addicts. Most often, such cases involve intoxication with benzodiazepines, Zopiclon, Zolpidem and diphenhydramine. Barbiturates and chloral hydrate are of minor significance today.

The clinical picture of intoxication with benzodiazepines, Zopiclon and Zolpidem is typically characterised by their depressive effect on the central nervous system. Depending on the dose, the patient develops a disturbance of consciousness ranging from somnolence to a reactive coma. In severe cases of benzodiazepine intoxication, especially in combination with alcohol, the patient may develop respiratory insufficiency that requires mechanical ventilation in combination with hypotension that necessitates the administration of catecholamines.

By contrast, diphenhydramine intoxication also leads to sedation, but there is a preponderance of symptoms of an anticholinergic syndrome with severe psychomotor agitation in conjunction with a strong jumpiness and the occurrence of visual hallucinations. In the case of serious diphenhydramine intoxication, these problems may also progress to agitated coma with cerebral seizures. To the clinical picture of diphenhydramine intoxication one must add peripheral anticholinergic symptoms such as tachycardia, hypertension, mydriasis and dryness of the skin and mucous membranes.

Therapeutic interventions on barbiturate intoxication, the therapeutic interventions focus firstly on stabilising the vital signs. With insufficient spontaneous breathing, endotracheal intubation is required, followed by mechanical ventilation. Hypotension in connection with severe barbiturate intoxication is initially treated by giving the patient fluids and, if this does not work, dopamine intravenously.

Specific antidotes are available for benzodiazepines (flumazenil) and for diphenhydramine (physostigmine salicylate). The initial dose of flumazenil for children is $0.01 \mathrm{mg} / \mathrm{kg}$ (up to $0.2 \mathrm{mg}$ ); adults are given an initial dose of flumazenil of $0.2 \mathrm{mg}$. However, the antagonising effect

Table 4 Important online sources of information.

\begin{tabular}{lll}
\hline Organisation & Abbreviation & URL \\
\hline Am Academy Clin Toxicology & AACT & ww.clintox.org \\
Am Academy Clinical Chemistry & AACC TDM/CT & www.acb.org.uk \\
Ass Clinical Biochemists & ACB & www.eapcct.org \\
Eur Ass Poisons Centers Clin Tox & EAPCCT & e.g., www.toxinfo.org \\
Poison information centre reports & & www.gtfch.org \\
Society of Toxicological and Forensic Chemistry & GTFCH & www.iatdmct.org \\
Int Ass Therapeutic Drug Mon Clin Tox & IATDMCT & www.ifcc.org/ifcc.asp \\
Int Fed Clin Chemistry Lab Medicine & IFCC & www.who.int/ipcs \\
Int Programme Chem Safity INTOX & IPCS, INTOX & www.soft-tox.org \\
Society Forensic Toxicologists & SOFT & www.tiaft.org \\
The Int Ass Forensic Toxicologists & TIAFT & \\
\hline
\end{tabular}


lasts only a short time, so that the injection is usually repeated or replaced by a permanent infusion with flumazenil. For specific therapy of the anticholinergic syndrome, physostigmine salicylate is administered in cases of diphenhydramine intoxication. The dose for children is $0.02-0.06 \mathrm{mg} / \mathrm{kg}$ (maximum $0.5 \mathrm{mg}$ ), adults are generally given $2 \mathrm{mg}$ of physostigmine salicylate.

The most common complications in barbiturate intoxications are pulmonary aspiration and the forming of a compartment syndrome. In benzodiazepine-addicted patients and in cases of mixed intoxication involving benzodiazepines and anticholinergically acting drugs (tricyclic antidepressants, diphenhydramine), antidote therapy with flumazenil can trigger cerebral seizures.

The above-described sedatives are captured by "general unknown" screening and can be quantified in the blood if necessary.

Poisoning with tricyclic antidepressants Psychiatric drug intoxications are observed primarily in the context of suicide attempts. Toxicologically, tricyclic antidepressants (TCA) play by far the biggest role. TCA intoxication affect primarily the central nervous system, the cardiovascular system and the vegetative nervous system. The effects on the central nervous system are initially similar to the symptoms of barbiturate intoxication and can range from somnolence to a reactive coma. Crucial for the further course of a TCA intoxication is the cardiotoxic effect of tricyclic antidepressants. TCA mainly lead to an inhibition of the rapid sodium influx in the myocardium with a resultant delay in the depolarisation of the myocardial cell membrane. In the ECG these changes initially lead to a widening of the QRS complex and to a prolongation of the QT-time. In cases of severe TCA intoxication, this stimulus conduction impairment then triggers supraventricular and ventricular arrhythmias, which in the absence of an adequate therapy can eventually result in ventricular tachycardia and ventricular fibrillation. Regardless of these arrhythmias, in severe TCA intoxications myocardial contractility is also reduced, accompanied by a sometimes critical drop in the arterial blood pressure. Finally, the clinical picture of a TCA intoxication also includes the effects on the vegetative nervous system in connection with an anticholinergic syndrome. The central anticholinergic effect manifests itself in hallucinations, cerebral seizures and the occurrence of an agitated coma. The peripheral anticholinergic symptoms include tachycardia, mydriasis as well as dry skin and mucous membranes.

The treatment of severe TCA intoxication focuses first on the stabilisation of vital parameters, with the treatment of cardiac arrhythmias being given top priority. An experimental therapy with antiarrhythmics of classes IA and IC may be fatal for the patient. Similar to the TCAs, antiarrhythmics of classes IA and IC also contribute to an inhibition of the rapid sodium influx in the myocardial cell membrane, i.e., the antiarrhythmics actually increase the cardiotoxic effect of the TCA further. As for a specific drug therapy of TCA-induced cardiac arrhythmias, the use of 1-2 mval/kg sodium bicarbonate has proved to be helpful in triggering an accelerated reactivation of the rapid sodium channels as well as increased protein binding of TCA (side effect: hypokalaemia). A therapeutic trial with physostigmine salicylate, which can reduce the heart rate and affect favourably the TCA-related inhibition of the rapid sodium influx, is rather risky, especially when the TCA-induced arrhythmia has already become bradycardiac in nature. Then, a further decrease in the heart rate may be provoked that could result in asystole.

The tricyclic antidepressants can be detected in the serum/plasma by means of immunochemical group tests. Only if the ingested substance and immunochemical cross-reactivity are precisely known, it is possible to provide a rough estimate of the plasma concentration. But this only applies to the traditional tricyclic antidepressants (amitriptyline, nortriptyline, doxepin and imipramine). The immunochemical result corresponds approximately to the sum of the concentrations of the respective medicinal substance and its metabolites (e.g., amitriptyline plus nortriptyline). Chromatographic determination of individual substances, e.g., by means of commercially available HPLC kits, is more reliable.

Poisoning with paracetamol Paracetamol (acetaminophen) is a very commonly used analgesic. In the case of an overdose from $200 \mathrm{mg} / \mathrm{kg}$, i.e., in adults from $10 \mathrm{~g}$ of paracetamol, a fulminant and life-threatening hepatonecrosis may occur. This is caused by a cytochrome P450-catalysed metabolic activation, which creates a highly reactive metabolite ( $\mathrm{N}$-acetyl-p-benzoquinone imine). This metabolite is also formed when therapeutic doses are ingested, but then is immediately detoxified by reaction with glutathione. In the case of paracetamol intoxication, however, the detoxification capacity may be exceeded. Acetyl cysteine is a very effective antidote, which can quickly replenish the body's own depleted glutathione stores. The administration of the antidote is certainly successful in treating paracetamol poisoning if it is given within the first $10 \mathrm{~h}$ after ingestion [47].

The hepatotoxic effect of paracetamol begins with a latency of approximately $24 \mathrm{~h}$, so that the plasma/serum analysis is crucial for suspected paracetamol intoxication. Such analysis can be done immunochemically (same methods as for therapeutic drug monitoring). If an adult is suspected of paracetamol poisoning, the following approach is recommended:

- Taking of blood sample for the determination of paracetamol, prothrombin time and/or quick test (INR), ALT, AST, creatinine, bilirubin, blood gases.

- When a patient is admitted for a suspected toxic dose, immediately begin antidote therapy ( $\mathrm{N}$-acetyl cysteine per infusion).

- Antidote treatment may be terminated if the paracetamol concentration was at any time below the treatment threshold. 
- Otherwise, continue antidote therapy for over $20 \mathrm{~h}$ and then repeat above blood tests. If the patient is asymptomatic and the test results are inconspicuous, it can be assumed that there will be very little risk for the patient, if any at all.

Even after more than $15 \mathrm{~h}$ between ingestion and the start of the antidote therapy, the survival rate for severe paracetamol intoxication is still better than it would be without the antidote and nearly as high as in the case of a liver transplant (60\%).

In terms of prognosis, a prothrombin time of up to $80 \mathrm{~s}$ is considered favourable; the survival rate is high up to $120 \mathrm{~s}$, but only $20 \%$ for $120 \mathrm{~s}$ and up. If fulminant hepatopathy has already occurred, the ratio between the coagulation factors $\mathrm{VII} / \mathrm{V}>30$ has a positive predictive value of $100 \%$ [47].

Especially in children, the paracetamol concentration should always be measured before any administration of the antidote, so that the use of the antidote can be medically validated.

Poisoning with antiarrhythmics The typical complications of intoxication with antiarrhythmics are hypotension and severe cardiac arrhythmias, which can manifest themselves already in the early stages of poisoning. Moreover, most of these arrhythmias must not be treated with the usual antiarrhythmics. The early detection, a diagnostic classification, as well as a targeted therapy of these arrhythmias are indispensable for the successful treatment of patients with antiarrhythmics intoxication. Most antiarrhythmics can be detected chromatographically in urine; quantification requires special methods.

Smoke gas intoxication In general, smoke gases are a heterogeneous mixture of substances. Its composition depends on the material burnt, the temperature and the supply of oxygen. Leading substances are carbon monoxide, hydrogen cyanide, hydrogen chloride and formaldehyde. During special burning events, other irritant gases may occur as well, such as nitrous gases, sulphur dioxide, acrolein, phosgene, ammonia or hydrogen fluoride.

Carbon monoxide exposure is detected by the formation of $\mathrm{COHb}$; $\mathrm{HCN}$ is found in exhaled air and in the blood (special test with a gas detection tube).

Intoxication with alkyl phosphates Alkyl phosphates are used as insecticides, with the most common products being ethyl parathion $\left(\mathrm{E}-605^{\circledR}\right)$, oxydemeton methyl (Metasystox $\mathrm{R}^{\circledR}$ ) and dimethoate $\left(\right.$ Roxion $\left.^{\circledR}\right)$. Although alkyl phosphates are absorbed easily by inhalation and percutaneously, severe life-threatening poisoning, however, has been observed only in cases where the toxins were ingested orally (important exception: nerve gas agent poisonings). Alkyl phosphates inhibit acetylcholinesterase (AChE), which creates a surplus of acetylcholine in the synapses of the autonomic and central nervous system as well as in the area of the neuromuscular endplate.

In lab tests, a strongly reduced plasma $\mathrm{CHE}$ is a good indicator of exposure to alkyl phosphates. The measurement of acetylcholinesterase in erythrocytes is necessary to verify an enzyme reactivation triggered by the antidote (atropine and oxime therapy). Detecting the substance requires specific procedures. The treatment is divided into primary care by stabilising the vital signs, antidote therapy, poison removal, intensive inpatient therapy with continuation of the antidote therapy (atropine) and symptom-based treatment of impaired organ functions. Atropine may be supplemented with further specific therapy (oxime therapy). Although atropine inhibits competitively the effect of acetylcholine at the muscarinic receptors, it has no influence on the nicotinic receptors on the motor endplate. Oximes, however, are a causally acting antidote, which involves the reactivation of the inhibited AChE. But reactivation is only possible as long as the AChE has not been inhibited irreversibly. This process, also referred to as "aging", functions at different rates, depending on the chemical structure of the organophosphate. The half-life of this "aging" is a few minutes for the combat agent Soman, several hours for oxydemetonmethyl and several days for parathion. What is crucial to the success of therapy, therefore, is that the oxime therapy must be started as early as possible. This therapy should be continued until either the inhibitive activity has disappeared in the serum, i.e., the level of plasma cholinesterase rises again, or until there is an aging of the erythrocytic AChE and, thus, the neuromuscular function continues to deteriorate despite the oxime therapy. The main indication for oxime therapy are intoxications with diethyl alkyl phosphates.

Amanita poisoning The accidental ingestion of amanita mushrooms leads to the so-called phalloides syndrome and is the most common cause of fatal mushroom poisoning. The amatoxins can be detected in urine by means of ELISA. The daily determination of creatinine and the Quick value from the third day of poisoning allow for a prognosis: Patients with a Quick $<25 \%$ and simultaneous creatinine $>1.2 \mathrm{mg} / \mathrm{dL}$ must undergo a liver transplant, while patients with a Quick $\geq 25 \%$ and creatinine $\leq 1.2 \mathrm{mg} / \mathrm{dL}$ definitely do not need a liver transplant. All non-classificable patients may need a liver transplant and remain in a readiness status for a transplant until they can be assigned to one of the two groups [48].

Poisoning with methanol or ethylene glycol Methanol and ethylene glycol form highly toxic metabolites. Therefore, these substances should be tested for in emergencies according to NACB recommendation [8], which is often not feasible. Alternatively, proof of metabolic acidosis with osmotic gap (methanol) or of calcium oxalate dihydrate crystals or in case of ethylene glycol by urine fluorescence from additional fluorescent dyes in anti- 
freeze products can be provided with substantial losses in sensitivity and specificity.

Poisoning with cyanide or sulphide The emergency physician must, on the basis of the circumstances and clinical tests, decide whether an antidote treatment is appropriate. It is recommended that blood samples be preserved for subsequent cyanide or sulphide analyses. Blood gas analysis on arterial and venous blood may help with an unclear decision-making situation. Low oxygen utilisation by tissue with lactic acidosis may indicate a more severe intoxication [8].

Intoxication by drugs of abuse Drug intoxication in the context of drug addiction usually involves an accidental overdose, but it is very difficult to distinguish between accidental or parasuicidal/suicidal drug intake. Most of these overdoses are observed in patients with a pronounced polytoxicomanic drug abuse, whereby not only one type of drug but several different drugs are consumed. The most common types currently involve heroin, methadone, benzodiazepines, alcohol and doxepin, all of which have a sedating effect on the central nervous system. Sometimes these drugs are also combined with substances that stimulate the central nervous system, such as cocaine or amphetamines. Hallucinogenic drugs like LSD and mushrooms, however, are of lesser importance in drug emergencies.

Heroin intoxication The cardinal symptoms of heroin intoxication range from a disturbance of consciousness (somnolence to deep coma) to respiratory depression and miosis. With insufficient spontaneous respiration, patients are intubated and ventilated in primary care and an intravenous cannula is inserted. Complications usually occur only if the opiate antagonist naloxone is administered additionally or instead of intubation, because the heroin-addicted patient does not only wake up quickly, but also because he develops a sudden withdrawal syndrome (excitation state involving, e.g., self-removal of the tube, vomiting with a correspondingly high risk of pulmonary aspiration, resistance to necessary hospitalisation). The patient can be taken to hospital safely only after sedation, for example, with benzodiazepines.

Intoxication with amphetamines or cocaine The main effects of these drugs manifest themselves on the central nervous system and the cardiovascular system. As for the central nervous system, a state of psychomotor agitation is triggered that may progress, with increasing severity, to an agitated coma with cerebral seizures. There have also been repeated reports of subarachnoid haemorrhages. As concerns the cardiovascular system, the first sign is an increase in blood pressure and the heart rate. In severe cases of intoxication, there may also be ventricular arrhythmias, anginous problems and, although only in rare cases, myocardial infarction.
The treatment of such poisoning focuses initially on the stabilisation of the vital signs. Diazepam is the drug of first choice for sedating the patient. When the patient develops strong agitation with recurrent cerebral seizures, treatment with muscle relaxants may be indicated. If the cardiovascular symptoms cannot be treated sufficiently with diazepam, Urapidil or nitrates can be administered. In the event of cocaine intoxication, the use of beta receptor blockers is generally advised against, because this could trigger an excessive stimulation of the alpha receptors followed by a resultant, aggravated vasoconstriction, thus reinforcing the symptoms of poisoning. Also, in treating arrhythmias brought on by cocaine, lidocaine should only be used with caution, as it lowers the threshold of cerebral agitation further, which has already been lowered by cocaine.

Poisoning with GHB or GBL ("liquid ecstasy") NACB [8] does not recommend any analysis from a clinical-toxicological point of view, because the clinical aspects and progression are very characteristic and usually shorter than the turnaround time for the test and quantification. The situation is different, of course, when it involves forensic aspects.

Mixed-drug intoxications These are common and there may be complications as part of an antidote therapy, which can pose a considerable problem both for primary care as well as for the transport of patients. A very common mixed intoxication in polytoxicomanic patients is the mixed intoxication resulting from heroin, benzodiazepines and alcohol, accompanied by loss of consciousness. If an opioid-addicted patient with mixed intoxication is treated with naloxone as part of antidote therapy, he or she will develop an acute withdrawal syndrome, which also involves, almost always, vomiting. The consciousness disturbance, which is possibly mostly caused by the simultaneous ingestion of benzodiazepines and alcohol, however, will be unaffected by the naloxone treatment. The result of such an antidote therapy is then a patient whose disturbance of consciousness continues, possibly accompanied by impaired cough and swallowing reflexes and simultaneous vomiting triggered by the opiate withdrawal. If the patient still had sufficient spontaneous breathing up to this point, then this constellation would almost inevitably lead to gastric contents being aspirated into the lung, producing acute respiratory insufficiency.

Another, increasingly frequently occurring drug intoxication found in polytoxicomanic patient lately has been the mixed use of heroin and cocaine. To intensify the desired euphoric effect, relatively high doses are consumed without causing serious side effects, because the side effects of heroin and cocaine on the central nervous system, at least, partially offset each other. If such a patient in the context of primary care is given naloxone, the sedating effect of heroin on the central nervous system is abolished, leading to an excessive reaction to 
cocaine. This can then further be complicated considerably by the symptoms of a severe cocaine intoxication, such as cerebral seizures, uncontrolled hypertension or cardiac arrhythmias. A similar outcome can be observed if a patient who has mixed cocaine and benzodiazepines is given flumazenil.

\section{Conclusions}

Despite major advances in instrumental analysis and clinical management of poisoned patients as well as in toxicological counselling, adequate timely toxicological analysis is still a very difficult issue [1].

The importance of toxicological analysis for emergency medicine cannot be evaluated definitively, for example, in terms of evidence-based medicine, since data from the literature are scarce and there are no available data outside of specialised centres. Therefore, recommendations from the literature $[1,8]$ and by professional associations should be followed that provide for a two-stage approach with emergency analyses in hospital laboratories and call for more complex analytical-toxicological procedures in specialist laboratories. For this purpose it is recommended that, if not already available, regional centres for toxicological analysis be set up and/or expanded.

Particularly in light of the very scarce financial resources and a very efficient (economical) use of staff, appropriate clinical-toxicological services will be feasible, or expanded further, only through regional centres. This raises the question of whether hospitals without such an integrated centre should conduct any toxicological analysis at all, which, to borrow from Gibitz, could be termed "first-line-analysis" [41]. Even though this initial analysis may not be particularly effective, it still serves important functions: The tests for a certain few poisons belong in an acute care hospital because of the quick turnaround time that is required. Almost more importantly, only through dealing with intoxication cases at the local laboratory, the staff can develop a level of sensitivity to deal with the subject matter and to seek out regional centres for further intoxication analysis, while continuously optimising the organisational conditions (sample preparation, primary advice for the clinician, transport of samples and communication of findings). Only through such a close contact and regular training can a high standard be achieved and maintained.

\section{References}

1. Flanagan RJ. Developing an analytical toxicology service: principles and guidance. Toxicol Rev 2004;23:251-63.

2. Proudfoot AT. Clinical assessment of the poisoned patient general principles. 12th Course on advances in Clinical Toxicology 1996, Birmingham, United Kingdom.

3. Arnold W. Cooperation between clinical intensive care units with forensic and toxicologic laboratories of institutes for forensic medicine in the treatment of acute poisoning. Anaesthesist 1969;18:353-8.

4. Free $A H$, Free HM. Urinalysis, critical discipline of clinical science. CRC Crit Rev Clin Lab Sci 1972;3:481-531.

5. Wallace JE, Blum K, Singh JM. Determination of drugs in biological specimens - a review. Clin Toxicol 1974;7:47795.

6. Hepler BR, Sutheimer CA, Sunshine I. The role of the toxicology laboratory in emergency medicine. Clin Toxicol 1982;19:353-65.

7. Hepler B, Sutheimer C, Sunshine I. Role of the toxicology laboratory in suspected ingestions. Pediatr Clin North Am 1986;33:245-60.

8. Wu AH, McKay C, Broussard LA, Hoffman RS, Kwong TC, Meyer TP, et al. National academy of clinical biochemistry laboratory medicine practice guidelines: recommendations for the use of laboratory test to support poisoned patients who present to the emergency department. Clin Chem 2003:49:357-79.

9. Külpmann WR, editor. Klinisch-toxikologische Analytik Verfahren, Befund, Interpretation. Weinheim (Germany): Whiley-VCH, 2002.

10. Külpmann WR, editor. Clinical toxicological analysis. Procedures, results, interpretation. Weinheim (Germany): Whiley-VCH, 2009.

11. Fukumoto M. Analytical role in clinical toxicology - impact on the diagnosis and treatment of a poisoned patient. Rinsho Byori 2008;56:330-4.

12. Baud FJ, Megarbane B, Deye N, Leprince P. Clinical review: aggressive management and extracorporeal support for drug-induced cardiotoxicity. Crit Care 2007;11:207.

13. Flanagan RJ, Widdop B, Ramsey JD, Loveland M. Analytical Toxicology. Hum Toxicol 1988;7:489-502.

14. Gareri J, Klein J, Koren G. Drugs of abuse testing in meconium. Clin Chim Acta 2006;366:101-11.

15. Schönberg L, Grobosch T, Lampe D, Kloft C. Toxicological screening in urine: comparison of two automated HPLC screening systems, toxicological identification system (TOX.I.S) versus REMEDI-HS. J Anal Toxicol 2007;31:321-7.

16. Maurer $\mathrm{HH}$. Systematic toxicological analysis procedures for acidic drugs and/or metabolites relevant to clinical and forensic toxicology and/or doping control. J Chromatogr B Biomed Sci Appl 1999;733:3-25.

17. Maurer $\mathrm{HH}$. Screening procedures for simultaneous detection of several drug classes used for high throughput toxicological analyses and doping control. A review. Comb Chem High Throughput Screen 2000;3:467-80.

18. Hallbach J. A fast and sensitive method for GC-MS screening in acute poisoning. Clin Tox 2003;41:558.

19. Maurer $\mathrm{HH}$. Position of chromatographic techniques in screening for detection of drugs or poisons in clinical and forensic toxicology and/or doping control. Clin Chem Lab Med 2004;42:1310-24.

20. Maurer HH. Hyphenated mass spectrometric techniques indispensable tools in clinical and forensic toxicology and in doping control. Mass Spectrum 2006;41:1399-413.

21. Maurer HH. Role of gas chromatography - mass spectrometry with negative ion chemical ionization in clinical and forensic toxicology, doping control, and biomonitoring. Ther Drug Monit 2002;24:247-54.

22. Marquet $P$, Lachatre G. Liquid chromatography - mass spectrometry: potential in forensic and clinical toxicology. Chromatogr B Biomed Sci Appl 1999;733:93-118.

23. Maurer $\mathrm{HH}$. Current role of liquid chromatography-mass spectrometry in clinical and forensic toxicology. Anal Bioanal Chem 2007;388:1315-25. 
24. Valli A, Polettini A, Papa P, Montagna M. Comprehensive drug screening by integrated use of gas chromatography/ mass spectrometry and Remedi HS. Ther Drug Monit 2001;23:287-94.

25. Hallbach J, Guder WG. Fast diagnosis of acute intoxications by a combined laboratory strategy of HPLC and GC-MS after ultrasonic derivatisation. Tth Alps-Adria Congress Regensburg 2002:108.

26. Müller CA, Weinmann W, Dresen S, Schreiber A, Gergov M. Development of a multi-target screening analysis for 301 drugs using a QTrap liquid chromatography/tandem mass spectrometry system and automated library searching. Rapid Commun Mass Spectrometr 2005;19:1332-8.

27. Pelander A, Ristimaa J, Rasanen I, Vuori E, Ojanperä I. Screening for basic drugs in hair of drug addicts by liquid chromatography/time-of-flight mass spectrometry. Ther Drug Monit 2008;30:717-24.

28. Bakdash A, Meyer-von A, Pragst F, Hallbach J. Detection of metabolites of selected psychoactive drugs in systematic toxicological analysis by LC-ESI-MS-TOF. Submitted 2009.

29. Megarbane B, Baud FJ. Interest of toxicological analysis for poisonings. Rev Prat 2008;58:838-43.

30. Hammett-Stabler CA, Pesce AJ, Cannon DJ. Urine drug screening in the medical setting. Clin Chim Acta 2002;315: 125-35.

31. Heyerdahl F, Hovda KE, Bjornaas MA, Brors O, Ekeberg O, Jacobsen D. Clinical assessment compared to laboratory screening in acutely poisoned patients. Hum Exp Toxicol 2008;27:73-9.

32. Helland A, Espnes KA, Reimers A, Aamo T, Zahlsen K, Rygnestad T, Spigset O. Toxicological screening of medicines and drugs of abuse in emergency cases. Tidsskr Nor Laegeforen 2008;128:42-5.

33. Epstein FB, Hassan M. Therapeutic drug levels and toxicology screen. Emerg Med Clin North Am 1986;4:367-76.

34. Tournier M, Molimard M, Titier K, Cougnard A, Begaud B, Gbikpi-Benissan G, et al. Accuracy of information on substance use recorded in medical charts of patients with intentional drug overdose. Psychiatr Res 2007;152:73-9.
35. Sugarman JM, Rodgers GC, Paul RI. Utility of toxicology screening in a pediatric emergency department. Pediatr Emerg Care 1997;13:194-7.

36. Belson MG, Simon HK. Utility of comprehensive toxicological screens in children. Am J Emerg Med 1999;17:221-4.

37. Hässler F, Zamorski H, Weirich S. The problem of differentiating between sudden infant death syndrome, fatal Munchhausen's syndrome by proxy, and infanticide. Z Kinder Jugendpsychiatr Psychother 2007;35:237-44.

38. Casavant MJ. Urine drug screening in adolescents. Pediatr Clin North Am 2002;49:317-27.

39. Proudfoot AT, Krenzelok EP, Vale JA. Position paper on urine alkalinization. J Toxicol Clin Toxicol 2004;42:1-26.

40. Hallbach J. The "diagnostic pathways in toxicology" project - a multidisciplinary approach. Clin Toxicol 2009 (invited keynote lecture at EAPCCT congress 2009).

41. Gibitz HJ. Emergency tests in the central laboratory of a hospital. Wien Med Wochenschr 1981;131:345-51.

42. Hallbach J, Guder WG. Mechanized toxicological serum tests in screening hospitalized patients. Eur J Clin Chem Clin Biochem 1991;29:537-47.

43. Kennedy M, Kiloh N. Drugs and brain death. Drug Saf 1996;14:171-80.

44. Hallbach J, von Meyer L, Maurer HH. Empfehlungen des Arbeitskreises Klinische Toxikologie der Gesellschaft für Toxikologische und Forensische Chemie (GTFCh) für die toxikologische Analytik im Rahmen der Hirntodfeststellung. $\mathrm{T}+\mathrm{K} 2002 ; 124-7$.

45. Heinzow BG, McLean A. Critical evaluation of current concepts in exposure assessment. Clin Chem 1994;40:136875.

46. Gil F, Pia A. Biomarkers as biological indicators of xenobiotic exposure. J Apl Toxicol 2001;21:245-55.

47. Vale JA. What is the optimum management of late presentation paracetamol poisoning. $12^{\text {th }}$ Course on advances in clinical toxicology. Birmingham 1996.

48. Ganzert M, Felgenhauer N, Zilker T. Indication of liver transplantation following amatoxin intoxication. $J$ Hepatol 2005;42:202-9. 